\title{
Frequency detuning effects for a parametric amplifier
}

Neumeyer, S.; Sorokin, V.S.; van Gastel, M. H. M.; Thomsen, J. J.

Published in:

Journal of Sound and Vibration

Link to article, DOI:

10.1016/j.jsv.2018.12.036

Publication date:

2019

Document Version

Peer reviewed version

Link back to DTU Orbit

Citation (APA):

Neumeyer, S., Sorokin, V. S., van Gastel, M. H. M., \& Thomsen, J. J. (2019). Frequency detuning effects for a parametric amplifier. Journal of Sound and Vibration, 445, 77-87. https://doi.org/10.1016/j.jsv.2018.12.036

\section{General rights}

Copyright and moral rights for the publications made accessible in the public portal are retained by the authors and/or other copyright owners and it is a condition of accessing publications that users recognise and abide by the legal requirements associated with these rights.

- Users may download and print one copy of any publication from the public portal for the purpose of private study or research.

- You may not further distribute the material or use it for any profit-making activity or commercial gain

- You may freely distribute the URL identifying the publication in the public portal

If you believe that this document breaches copyright please contact us providing details, and we will remove access to the work immediately and investigate your claim. 


\section{Accepted Manuscript}

Frequency detuning effects for a parametric amplifier

S. Neumeyer, V.S. Sorokin, M.H.M. van Gastel, J.J. Thomsen

PII: $\quad$ S0022-460X(18)30862-9

DOI: $\quad$ https://doi.org/10.1016/j.jsv.2018.12.036

Reference: $\quad$ YJSVI 14572

To appear in: Journal of Sound and Vibration

Received Date: 30 August 2018

Revised Date: 22 November 2018

Accepted Date: 28 December 2018

Please cite this article as: S. Neumeyer, V.S. Sorokin, M.H.M. van Gastel, J.J. Thomsen, Frequency detuning effects for a parametric amplifier, Journal of Sound and Vibration (2019), doi: https:// doi.org/10.1016/j.jsv.2018.12.036.

This is a PDF file of an unedited manuscript that has been accepted for publication. As a service to our customers we are providing this early version of the manuscript. The manuscript will undergo copyediting, typesetting, and review of the resulting proof before it is published in its final form. Please note that during the production process errors may be discovered which could affect the content, and all legal disclaimers that apply to the journal pertain. 


\title{
Frequency detuning effects for a parametric amplifier
}

\author{
S. Neumeyer ${ }^{\mathrm{a}}$, V. S. Sorokin ${ }^{\mathrm{c}}$, M. H. M. van Gastel ${ }^{\mathrm{b}}$, J. J. Thomsen ${ }^{\mathrm{a}}$ \\ ${ }^{a}$ Department of Mechanical Engineering, Solid Mechanics, \\ Technical University of Denmark, Denmark \\ ${ }^{b}$ Department of Mechanical Engineering, Dynamics and Control, \\ Eindhoven University of Technology, The Netherlands \\ ${ }^{c}$ Acoustics Research Centre, Department of Mechanical Engineering, The University of \\ Auckland, New Zealand
}

\begin{abstract}
Frequency tuned parametric amplifiers may experience changes in both the two-to-one frequency ratio between the parametric and the direct excitation, and between the direct excitation frequency and the systems natural frequency. These effects are investigated theoretically using a Duffing-Mathieu equation as the model system, and investigated experimentally using a macro cantilever beam as the model object. The approximate analytical steadystate vibration amplitudes are derived using the method of varying amplitudes, and compared with results of direct numerical integration, showing good agreement. Theoretical predictions reveal that for detuned superthreshold parametric amplification some of the amplitude-frequency curves appear to collapse. Experiments show that a drop in the maximum steady-state vibration amplitude occurs for specific areas in the amplitude-excitation detuning domain, whereas for other areas frequency detuning may yield an increased maximum steady-state vibration amplitude. Thus frequency detuning is a feature which can purposefully be avoided or utilized, dependent on the usage e.g. for sensors or energy harvesters. We report experimentally obtained bistable amplified steady-state responses, which also support theoretical findings.
\end{abstract}

Keywords: parametric amplification, frequency detuning, nonlinearity

\section{Introduction}

Parametric amplification (in the present context understood as adding parametric excitation to direct excitation for boosting resonant oscillations) 
may be exploited in mechanical [1, 2], electrical [3], optical [4], acoustical [5] and thermal [6] systems. It has been utilized for e.g. mass and force sensing, signal filtering, timing, switching and signal processing $[7,8,9]$. Recently parametric amplification was examined in a macromechanical context $[10,11]$ as a means for amplifying the output of resonant macrotransducers. The effect of superthreshold parametric pumping was demonstrated in [12]; here one adds parametric excitation to direct excitation, where the resulting steady-state vibration amplitude-frequency response is dominated by the parametric excitation component, and where the parametric instability threshold is associated with the Arnold tongue. Optimal excitation parameters, i.e. how to maximize the steady-state vibration amplitude for a superthreshold parametrically pumped amplifier, were examined in [13]. Effects of cubic nonlinearity on subthreshold parametrically pumped steady-state vibration amplitude and frequency bandwidth was examined theoretically in $[12,14,15]$ and experimentally in [16]. Most of these references consider a Duffing-Mathieu system. This is similar to [17] which has a reference to wind turbine blades, to [18] where the effect of quadratic and cubic nonlinearities are considered, and to [19] where higher-order resonances are studied.

It has been common to distinguish between perfectly tuned (i.e. a strict two-to-one frequency ratio between the principle parametric and the direct excitation) and detuned parametric amplification. In this context detuned parametric amplification was understood as a frequency ratio between the parametric and the direct excitation much different from two-to-one $[10,13]$. In the perfectly tuned case a steady-state vibration amplitude which is dependent on the excitation phase has been observed, and in the strongly detuned case one obtains a steady-state vibration amplitude which is independent on the excitation phase. The present work examines effects of frequency detuning close to the two-to-one ratio between the parametric and the direct excitation frequencies. This is motivated by changes in frequencies, where the two-to-one ratio between the parametric and the direct excitation, and also the closeness to resonance, might undergo slight variations during normal operation. The two-to-one frequency ratio could also be affected by e.g. uncertainties with respect to physically adding the parametric excitation component, and the natural frequency could change due to e.g. wear, damage, or varying mass loading.

As a mathematical model, a Duffing-Mathieu equation with independent frequencies for the parametric and direct excitation is considered. The steady-state vibration amplitude is predicted approximately using the method 
of varying amplitudes (MVA) [20], and compared to results of numerical direct integration and experimental observations. Compared to the preliminary work [21] the present paper provides elaborate theoretical predictions, details about the experimental setup, time-domain results, and additional experimental results in terms of amplitude-excitation phase and amplitudefrequency relations.

\section{Theoretical predictions}

A forced Duffing-Mathieu equation with parametric and direct excitation frequencies $\Omega_{p}$ and $\Omega_{d}$ is investigated:

$$
\ddot{x}+\beta \dot{x}+\omega_{0}^{2}\left(1+p \cos \left(\Omega_{p} t\right)\right) x+\gamma x^{3}=d \cos \left(\Omega_{d} t+\phi\right),
$$

where $\left({ }^{\circ}\right)$ denotes temporal derivatives, $\beta=2 \omega_{0} \zeta$ where $\zeta$ is the damping ratio, $\omega_{0}$ is the linear natural frequency, $\gamma$ the nonlinear coefficient, $p$ the parametric excitation amplitude, $d$ the direct excitation amplitude, $t$ time, and $\phi$ the phase between the parametric and direct excitation.

A simple system described by (1) is a beam with a vibrated suspension, as shown in Fig. 1, where horizontal (axial) vibrations correspond to parametric excitation, vertical (transverse) vibrations correspond to direct excitation, the displacement amplitudes $\tilde{p}$ and $\tilde{d}$ are proportional to the excitation coefficients $p$ and $d$ in (1), and $x$ represents the modal coefficient of a single vibration mode.

\subsection{General steady-state model response}

The frequencies $\Omega_{p}$ and $\Omega_{d}$ are considered constant in time or quasistatically varying, and are typically tuned to be commensurate for parametric

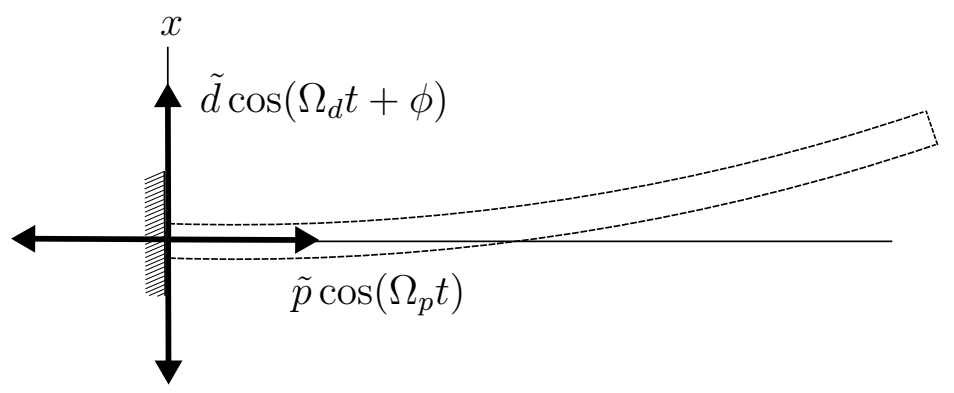

Figure 1: Schematic illustration of mathematical model (1). 
amplifiers, i.e. $\Omega_{p} / \Omega_{d}=2$, which provides a periodic steady-state response. Frequency detuning, by contrast, generally yields quasi-periodic steady-state responses. Note that conventional harmonic balance methods are not applicable to obtain solutions describing quasi-periodic responses, whereas methods based on Floquet thoery are only for linear pure periodic systems. Employing conventional asymptotic methods, e.g. the multiple scales perturbation method, for the considered problem results in non-autonomous nonlinear equations for amplitude and phase for the response, thus the MVA is used to solve Eq. (1). First a frequency detuning $\sigma_{p d}$ between the parametric and the direct excitation is introduced, defined by:

$$
\sigma_{p d}=\Omega_{d}-\frac{1}{2} \Omega_{p}
$$

quantifying the nearness to exact two-to-one tuning. The MVA assumes a solution form consisting of a series of harmonics with time-varying amplitudes $B_{m 1}$ and $B_{m 2}$ :

$$
x(t)=\sum_{m=1}^{n} B_{m 1}(t) \cos \left(\frac{1}{2} m \Omega_{p} t\right)+B_{m 2}(t) \sin \left(\frac{1}{2} m \Omega_{p} t\right),
$$

which, by contrast to perturbation methods such as multiple scales or averaging [22], is without restrictions such as slowly varying amplitudes. Also, by contrast to the method of harmonic balance where the coefficients $B_{m 1}$ and $B_{m 2}$ would be constants and Eq. (3) an approximation, the allowed time dependency of $B_{m 1}$ and $B_{m 2}$ means that Eq. (3) merely represents a shift of variables, which is exact for any value of $n$, even $n=1$. The shift from the original dependent variable $x$ to $2 n$ new variables, $B_{m 1}$ and $B_{m 2}$, implies that $2 n$ equations are needed. This can be accomplished by introducing constraints in the form of $2 n-1$ additional equations. The constraints are introduced by substituting Eq. (3) into Eq. (1), and requiring $2 n-1$ groups of terms to equal zero. Equation $2 n$ includes all the remaining terms including the harmonics having order higher than $n$. The $2 n-1$ groups of terms are proposed to be the coefficients of the involved harmonic terms. Considering only the first harmonic terms in Eq. (3), i.e. $n=1$, one obtains two equations for the amplitudes $B_{1}$ and $B_{2}$ (in which the first index has been omitted since of no importance for $n=1$ ):

$$
\begin{aligned}
\ddot{B}_{1}+\beta \dot{B}_{1}+\Omega_{p} \dot{B}_{2}+\frac{1}{2} \beta \Omega_{p} B_{2}+ & \left(\omega_{0}^{2}\left(1+\frac{1}{2} p\right)-\frac{1}{4} \Omega_{p}^{2}\right) B_{1} \\
& +\frac{3}{4} \gamma\left(B_{1}^{2}+B_{2}^{2}\right) B_{1}=d \cos \left(\sigma_{p d} t+\phi\right),
\end{aligned}
$$




$$
\begin{aligned}
\ddot{B}_{2}+\beta \dot{B}_{2}-\Omega_{p} \dot{B}_{1}-\frac{1}{2} \beta \Omega_{p} B_{1} & +\left(\omega_{0}^{2}\left(1-\frac{1}{2} p\right)-\frac{1}{4} \Omega_{p}^{2}\right) B_{2} \\
& +\frac{3}{4} \gamma\left(B_{1}^{2}+B_{2}^{2}\right) B_{2}=-d \sin \left(\sigma_{p d} t+\phi\right) .
\end{aligned}
$$

Thus Eq. (1) is restated through eqs. (4)-(5), with $B_{1}$ and $B_{2}$ as the dependent variables instead of $x$. Approximations have been introduced by neglecting higher harmonics. This simplification is valid for small values of the nonlinear term $\gamma x^{3}$ and parametric excitation term $p \omega_{0}^{2} x$, compared to the linear restoring term $\omega_{0}^{2} x$. To solve eqs. (4) and (5) a shift of variables from $B_{1}$ and $B_{2}$ is performed:

$$
B_{1}(t)=B_{1 s}+B_{1 n s}(t), \quad B_{2}(t)=B_{2 s}+B_{2 n s}(t),
$$

where $B_{1 s}$ and $B_{2 s}$ are constants that satisfy:

$$
\begin{aligned}
\frac{1}{2} \beta \Omega_{p} B_{2 s}+B_{1 s}\left(\omega_{0}^{2}\left(1+\frac{1}{2} p\right)-\frac{1}{4} \Omega_{p}^{2}\right)+\frac{3}{4} \gamma B_{1 s}\left(B_{1 s}^{2}+B_{2 s}^{2}\right) & =0, \\
-\frac{1}{2} \beta \Omega_{p} B_{1 s}+B_{2 s}\left(\omega_{0}^{2}\left(1-\frac{1}{2} p\right)-\frac{1}{4} \Omega_{p}^{2}\right)+\frac{3}{4} \gamma B_{2 s}\left(B_{1 s}^{2}+B_{2 s}^{2}\right) & =0 .
\end{aligned}
$$

Substituting Eq. (6) into eqs. (4) and (5), and considering eqs. (7)-(8), one obtains equations for $B_{1 n s}$ and $B_{2 n s}$ :

$$
\begin{aligned}
\ddot{B}_{1 n s}+\beta \dot{B}_{1 n s}+ & \Omega_{p} \dot{B}_{2 n s}+\frac{1}{2} \beta \Omega_{p} B_{2 n s}+\left(\omega_{0}^{2}\left(1+\frac{1}{2} p\right)-\frac{1}{4} \Omega_{p}^{2}\right) B_{1 n s} \\
& +\frac{3}{4} \gamma\left[B_{1 n s}\left(3 B_{1 s}^{2}+B_{2 s}^{2}+3 B_{1 s} B_{1 n s}+B_{1 n s}^{2}\right)\right. \\
& \left.+\left(2 B_{2 s}+B_{2 n s}\right)\left(B_{1 s}+B_{1 n s}\right) B_{2 n s}\right]=d \cos \left(\sigma_{p d} t+\phi\right), \\
\ddot{B}_{2 n s}+\beta \dot{B}_{2 n s}- & \Omega_{p} \dot{B}_{1 n s}-\frac{1}{2} \beta \Omega_{p} B_{1 n s}+\left(\omega_{0}^{2}\left(1-\frac{1}{2} p\right)-\frac{1}{4} \Omega_{p}^{2}\right) B_{2 n s} \\
& +\frac{3}{4} \gamma\left[B_{2 n s}\left(3 B_{2 s}^{2}+B_{1 s}^{2}+3 B_{2 s} B_{2 n s}+B_{2 n s}^{2}\right)\right. \\
+ & \left.\left(2 B_{1 s}+B_{1 n s}\right)\left(B_{2 s}+B_{2 n s}\right) B_{1 n s}\right]=-d \sin \left(\sigma_{p d} t+\phi\right) .
\end{aligned}
$$

To solve eqs. (9)-(10) one can employ the MVA once more and search for a solution in the form:

$$
B_{k n s}=C_{k 1}(t) \cos \psi+C_{k 2}(t) \sin \psi,
$$

where $\psi=\sigma_{p d} t+\phi$ and $k=1,2$. This gives the following equations for $C_{k 1}=C_{k 1}(t)$ and $C_{k 2}=C_{k 2}(t)$ :

$$
\begin{aligned}
\gamma\left[\frac{3}{4}\left(3 B_{1 s}^{2}+B_{2 s}^{2}+\frac{3}{4} C_{11}^{2}+\frac{3}{4} C_{12}^{2}+\frac{3}{4} C_{21}^{2}+\frac{1}{4} C_{22}^{2}\right) C_{11}\right. \\
\left.+\frac{3}{2}\left(\frac{1}{4} C_{12} C_{22}+B_{1 s} B_{2 s}\right) C_{21}\right]+\left(\omega_{0}^{2}+\frac{1}{2} p \omega_{0}^{2}-\frac{1}{4} \Omega_{p}^{2}-\sigma_{p d}^{2}\right) C_{11} \\
+\frac{1}{2} \beta \Omega_{p} C_{21}+\beta \sigma_{p d} C_{12}+\sigma_{p d} \Omega_{p} C_{22}+\beta \dot{C}_{11} \\
+2 \sigma_{p d} \dot{C}_{12}+\Omega_{p} \dot{C}_{21}+\ddot{C}_{11}=d
\end{aligned}
$$




$$
\begin{gathered}
\gamma\left[\frac{3}{4}\left(3 B_{1 s}^{2}+B_{2 s}^{2}+\frac{3}{4} C_{11}^{2}+\frac{3}{4} C_{12}^{2}+\frac{1}{4} C_{21}^{2}+\frac{3}{4} C_{22}^{2}\right) C_{12}\right. \\
\left.+\frac{3}{2}\left(\frac{1}{4} C_{11} C_{21}+B_{1 s} B_{2 s}\right) C_{22}\right]+\left(\omega_{0}^{2}+\frac{1}{2} p \omega_{0}^{2}-\frac{1}{4} \Omega_{p}^{2}-\sigma_{p d}^{2}\right) C_{12} \\
+\frac{1}{2} \beta \Omega_{p} C_{22}-\beta \sigma_{p d} C_{11}-\sigma_{p d} \Omega_{p} C_{21}+\beta \dot{C}_{12} \\
-2 \sigma_{p d} \dot{C}_{11}+\Omega_{p} \dot{C}_{22}+\ddot{C}_{12}=0 \\
\gamma\left[\frac{3}{4}\left(B_{1 s}^{2}+3 B_{2 s}^{2}+\frac{3}{4} C_{11}^{2}+\frac{1}{4} C_{12}^{2}+\frac{3}{4} C_{21}^{2}+\frac{3}{4} C_{22}^{2}\right) C_{21}\right. \\
\left.+\frac{3}{2}\left(\frac{1}{4} C_{12} C_{22}+B_{1 s} B_{2 s}\right) C_{11}\right]+\left(\omega_{0}^{2}-\frac{1}{2} p \omega_{0}^{2}-\frac{1}{4} \Omega_{p}^{2}-\sigma_{p d}^{2}\right) C_{21} \\
-\frac{1}{2} \beta \Omega_{p} C_{11}+\beta \sigma_{p d} C_{22}-\sigma_{p d} \Omega_{p} C_{12}+\beta \dot{C}_{21} \\
+2 \sigma_{p d} \dot{C}_{22}-\Omega_{p} \dot{C}_{11}+\ddot{C}_{21}=0 \\
\gamma\left[\frac{3}{4}\left(B_{1 s}^{2}+3 B_{2 s}^{2}+\frac{1}{4} C_{11}^{2}+\frac{3}{4} C_{12}^{2}+\frac{3}{4} C_{21}^{2}+\frac{3}{4} C_{22}^{2}\right) C_{22}\right. \\
\left.+\frac{3}{2}\left(\frac{1}{4} C_{11} C_{21}+B_{1 s} B_{2 s}\right) C_{12}\right]+\left(\omega_{0}^{2}-\frac{1}{2} p \omega_{0}^{2}-\frac{1}{4} \Omega_{p}^{2}-\sigma_{p d}^{2}\right) C_{22} \\
-\frac{1}{2} \beta \Omega_{p} C_{12}-\beta \sigma_{p d} C_{21}+\sigma_{p d} \Omega_{p} C_{11}+\beta \dot{C}_{22} \\
-2 \sigma_{p d} \dot{C}_{21}-\Omega_{p} \dot{C}_{12}+\ddot{C}_{22}=-d,
\end{gathered}
$$

in which higher harmonics have been neglected. Results are valid for small values of the nonlinear term and parametric excitation term compared to the linear restoring term. Neglecting all time derivatives in eqs. (12)-(15), and solving for the constant amplitudes $C_{k 1}=C_{k 1 s}$ and $C_{k 2}=C_{k 2 s}$ (omitted for brevity), gives the steady-state approximate solution of Eq. (1):

$$
\begin{aligned}
x(t) & =\left(B_{1 s}+C_{11 s} \cos \left(\sigma_{p d} t+\phi\right)+C_{12 s} \sin \left(\sigma_{p d} t+\phi\right)\right) \cos \left(\frac{1}{2} \Omega_{p} t\right) \\
& +\left(B_{2 s}+C_{21 s} \cos \left(\sigma_{p d} t+\phi\right)+C_{22 s} \sin \left(\sigma_{p d} t+\phi\right)\right) \sin \left(\frac{1}{2} \Omega_{p} t\right) .
\end{aligned}
$$

\subsection{Perfectly tuned superthreshold steady-state response}

Fig. 2 depicts superthreshold parametrically pumped steady-state vibration amplitude as a function of direct excitation detuning $\left(\sigma_{p d}=0\right)$. The instability threshold associated with superthreshold pumping corresponds to primary parametric instability of the zero solution of the linearized Eq. (1) for the case of pure parametric excitation. Fig. 2(a) shows that adding superthreshold parametric pumping to a directly excited system yields a bistable amplified steady-state peak. Good agreement between the theoretical predictions and results of direct numerical integration is noted even for 

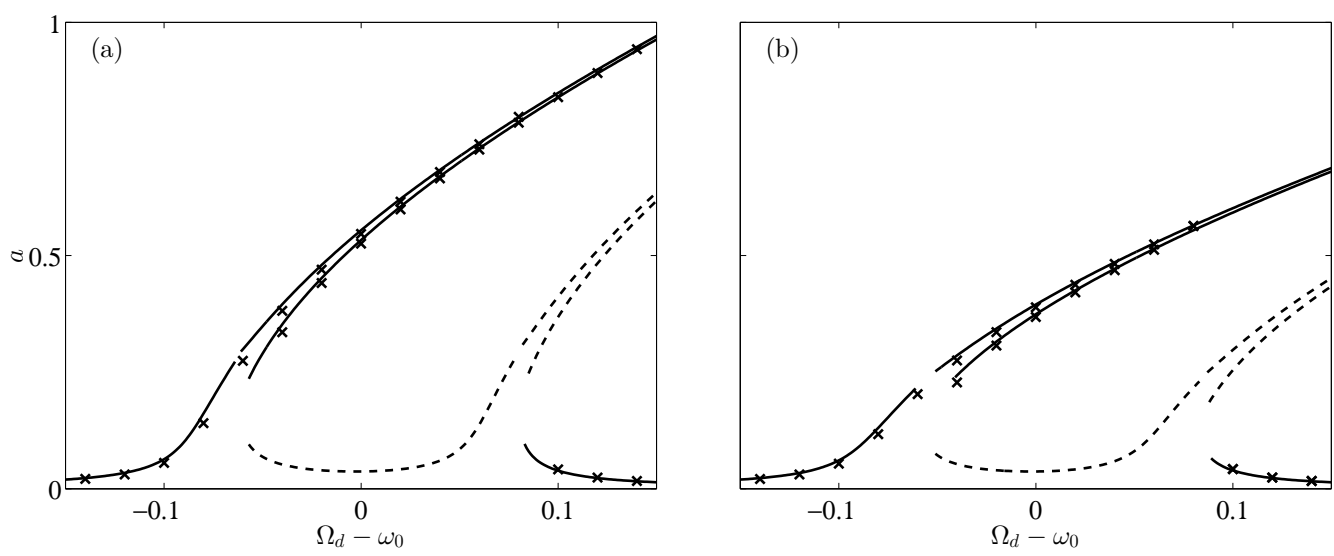

Figure 2: Theoretical superthreshold parametrically pumped steady-state vibration amplitude as a function of direct excitation detuning using the MVA ( - ) alongside results of direct numerical integration $(\times)$ for (a) $\gamma=0.05$ and (b) $\gamma=0.1$. Dashed lines denote an approximate analytical unstable response. $\left(\Omega_{p} / \Omega_{d}=2, \beta=0.1, \phi=-\pi / 4, p=0.35\right.$, $d=0.01, \omega_{0}=1$.)

not weak nonlinearity and parametric excitation. The gaps at the base of each peak can be reduced by introducing higher order harmonics into the solution. Results of direct numerical integration support this claim. A distinct feature, compared to the subthreshold parametrically pumped case is, that in the resonance frequency range the steady-state vibration amplitude increases significantly. This feature might be useful for sensor applications, because the transition from a small to a large amplitude becomes more pronounced, and it might also be useful for energy harvesters due to the larger amplitude. Fig. 2(b) shows that increasing the nonlinear coefficient $\gamma$ hardens the superthreshold parametrically pumped response, similarly to the case of a subthreshold nonlinear hardened response. In other words the impact of increasing the nonlinear coefficient is qualitatively the same for sub- and superthreshold parametric pumping. The values of parameters and meaning of markers and curves are given in the caption of Fig. 2 and used in subsequent figures unless stated otherwise.

\subsection{Detuned sub- and superthreshold steady-state response}

Fig. 3 depicts the sub- and superthreshold parametrically pumped steadystate vibration amplitude as a function of parametric excitation detuning $\left(\Omega_{p}-2 \omega_{0}\right.$ while $\left.\Omega_{d}=\omega_{0}\right)$. The inserts show typical time-responses. Beating is obtained in the detuned case. For $\Omega_{p}=2 \omega_{0}$ there is no beating as 
illustrated by the single circle without a solid vertical line in Fig. 3(a). Beating occurs in all results shown in Fig. 3(b) because none of these are strict $\Omega_{p}=2 \omega_{0}$. The nearest result $\Omega_{p}-2 \omega_{0} \approx 0.01$ and thus with beating. For subthreshold parametric pumping (Fig. 3(a)) the steady-state vibration amplitude is maximum slightly away from linear parametric resonance $\left(\Omega_{p}=2 \omega_{0}\right)$ due to the effect of cubic nonlinearity. This effect is pronounced in the case of superthreshold parametric pumping (Fig. 3(b)) until a jump happens. These results show that one can obtain high steady-state vibration amplitudes even in the nonlinear case with beating.

Fig. 4 shows detuned $\left(\sigma_{p d} \neq 0\right)$ superthreshold parametrically pumped steady-state vibration amplitudes. A frequency ratio $\Omega_{p} / \Omega_{d}=2.05$ and a frequency ratio $\Omega_{p} / \Omega_{d}=1.95$ between the parametric and the direct excitation are used. The somewhat larger deviation between the approximate analytical solution and results of direct numerical integration around the intersection between the lower solution and the stable peak, can be reduced by including higher harmonics in the approximation (i.e. $n>1$ in Eq. (3)), though omitted here since the calculations become more lengthy with only minor increase in accuracy. Up to five solution branches coexist at a given frequency. Two of them are duplicates in the steady-state amplitude-frequency relation except for a difference in excitation phase. That is, with frequency detuning between the parametric and direct excitation, the branches of the bistable amplified peak of Fig. 2 appear to collapse into a single curve. This is contrary to perfect frequency tuning (Fig. 2 and [12, 23, 24]). Jumps are observed both during a down- and upsweep in $\Omega_{d}$. The upsweep jump is, for the chosen parameters, considerably smaller than the downsweep jump, but this might be otherwise for other parameter values. Also, the frequency separation between the two peaks changes with the frequency detuning $\sigma_{p d}$ between the parametric and the direct excitation. Compared to the perfectly tuned case (Fig. 2), an upsweep jump emerges, and both peaks appear to shift to either the left or right side of linear resonance. Furthermore, slightly frequency detuned superthreshold parametric pumping can result in a further increase in steady-state vibration amplitude. For a negative nonlinear coefficient $\gamma$ the steady-state vibration amplitude bends to the left instead of to the right, and the peaks of the stable and unstable solutions interchange stability.

To summarize the results of figs. 2 and 4 , it is noted that with perfectly tuned subthreshold parametric pumping, the response qualitatively resembles that of a system subjected to pure direct excitation. The parametric 

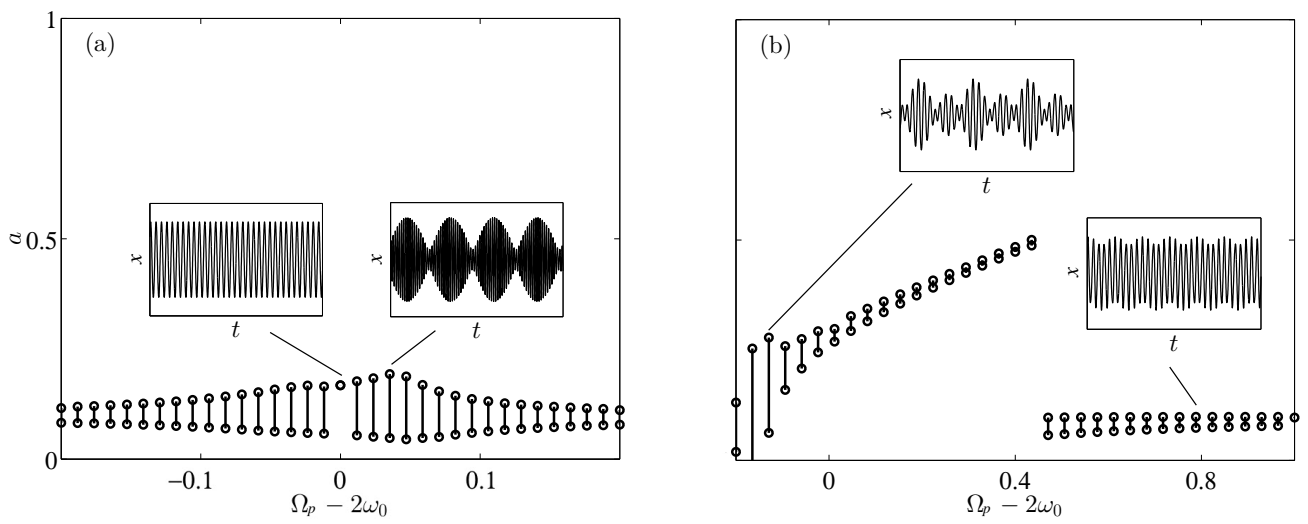

Figure 3: Numerical simulation of nonlinear (a) subthreshold $p=0.15$ and (b) superthreshold parametrically pumped $p=0.55$ steady-state vibration amplitude as a function of parametric excitation detuning $\left(\Omega_{p}-2 \omega_{0}\right.$ while $\left.\Omega_{d}=\omega_{0}\right)$. Solid vertical lines denote amplitude modulation ranges for beating responses, and circles maximum and minimum values. $(\gamma=4$. $)$

pumping component merely increases the steady-state vibration amplitude - mostly around the linear resonance frequency. With perfectly tuned superthreshold parametric pumping a bistable amplified steady-state response is observed. With detuned $\left(\Omega_{p} / \Omega_{d} \neq 1\right)$ subthreshold parametric pumping the steady-state response drops in the entire frequency range. With detuned superthreshold parametric pumping both characteristics of the pure directly excited system and of the pure parametrically excited system are observed. Specifically, the zero solution of a purely parametrically excited system is not present, but the abrupt amplitude-frequency shift, as present for a purely parametrically excited system, is observed. The nonzero solution on each side of the two peaks is due to the direct excitation component, and appears split into two due to the parametric excitation component. The responses that are split up becomes more nonlinear for increasing steady-state vibration amplitude due to the effect of the cubic stiffness nonlinearity. For a purely parametrically excited system with a hardening nonlinearity $(\gamma>0)$, the upper left (lower right) amplitude-frequency solution is stable (unstable); this is also the case for detuned superthreshold parametric pumping. It appears that not only is the parametric excitation component dominant in the case of perfectly tuned superthreshold parametric pumping, as also noticed in [12], but its characteristics become even more dominant with frequency 

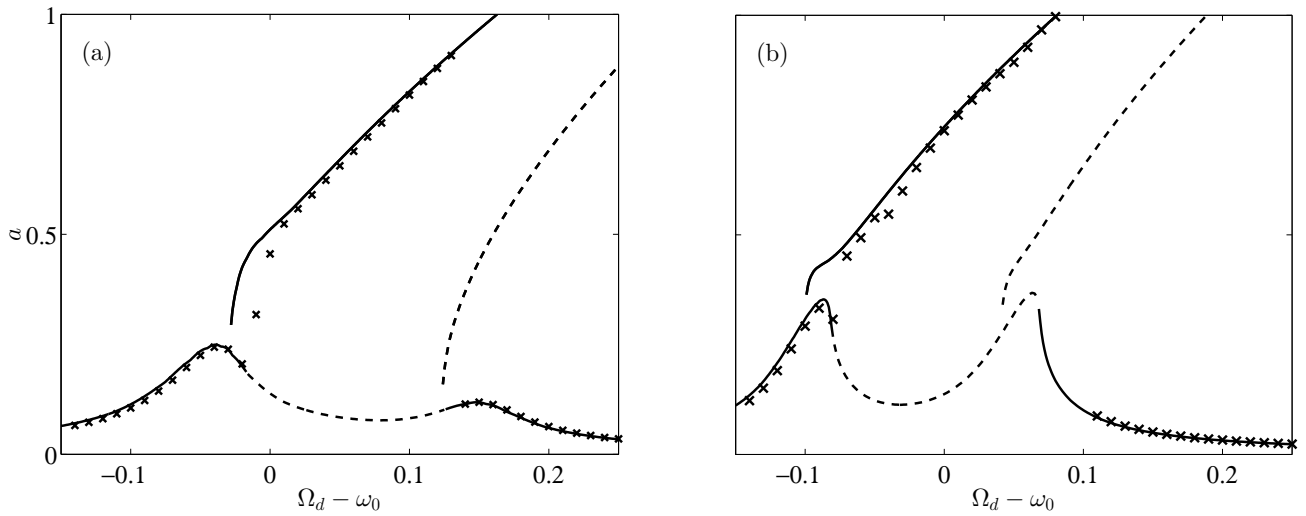

Figure 4: Theoretical superthreshold parametrically pumped steady-state vibration amplitude as a function of direct excitation detuning for (a) $\Omega_{p} / \Omega_{d}=2.05$ and (b) $\Omega_{p} / \Omega_{d}=1.95$. $(p=0.35, \gamma=1$. $)$

detuning.

Fig. 5 shows the steady-state vibration amplitude, using results obtained by numerical direct integration of Eq. (1), as a function of parametric and direct excitation frequencies for sub- and superthreshold parametric pumping. The magnitudes of the steady-state vibration amplitudes are given in the colourbars. They have been normalized with respect to the minimum value which yields parametric resonance, and not the maximum steady-state vibration amplitude to improve the scaling of figs. 5 and 6 . In the subthreshold parametrically pumped case (Fig. 5(a)), the steady-state vibration amplitude is mostly dependent on the direct excitation frequencies as opposed to the case of superthreshold parametric pumping (Fig. 5(b)). Interchanging the inner and outer loop, i.e. parametric and direct excitation frequency increments, yields identical steady-state vibration amplitudes, even though initial conditions from the previous steady-state solution are used. This is opposed to the experimental results discussed in Sect. 3.

\subsection{Dependency on the excitation phase}

Fig. 6 shows perfectly tuned $\left(\sigma_{p d}=0\right)$ sub- and superthreshold parametrically pumped steady-state vibration amplitudes as a function of excitation phase $\phi$ and direct excitation detuning, using results of direct numerical integration. With $\Omega_{d}=\omega_{0}$ figs. 6(a,b) simplify to typical two-dimensional steady-state vibration amplitude plots with the excitation phase as the de- 

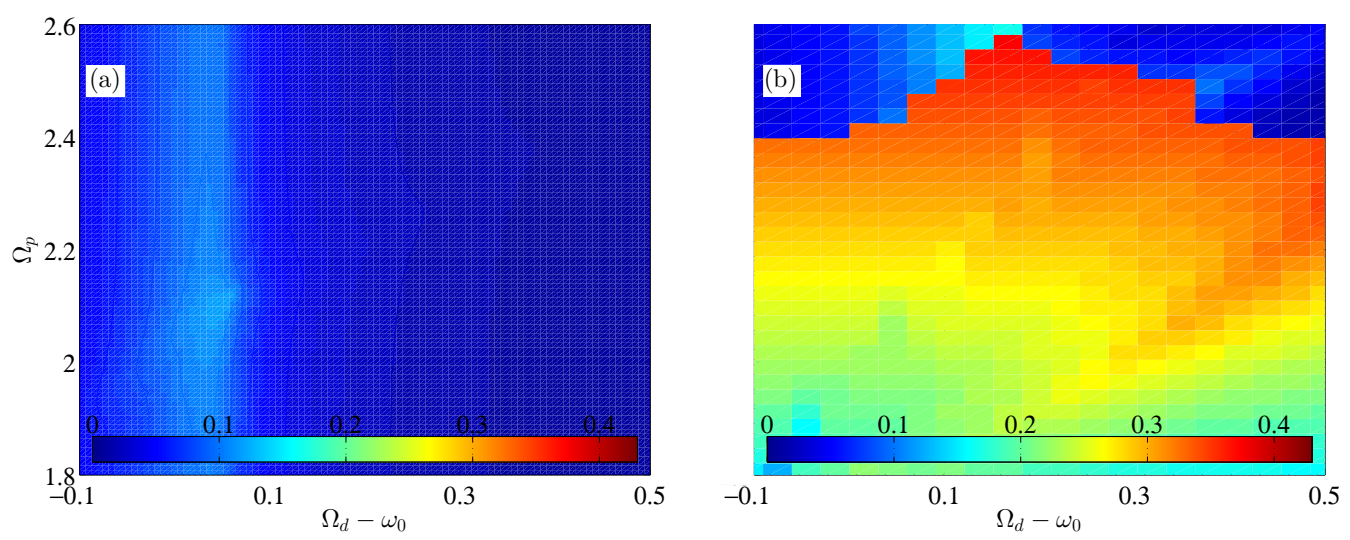

Figure 5: (a) subthreshold $p=0.04$ and (b) superthreshold $p=0.8$ parametrically pumped steady-state vibration amplitude as a function of direct and parametric excitation detuning using results of direct numerical integration. $(\gamma=10$. $)$
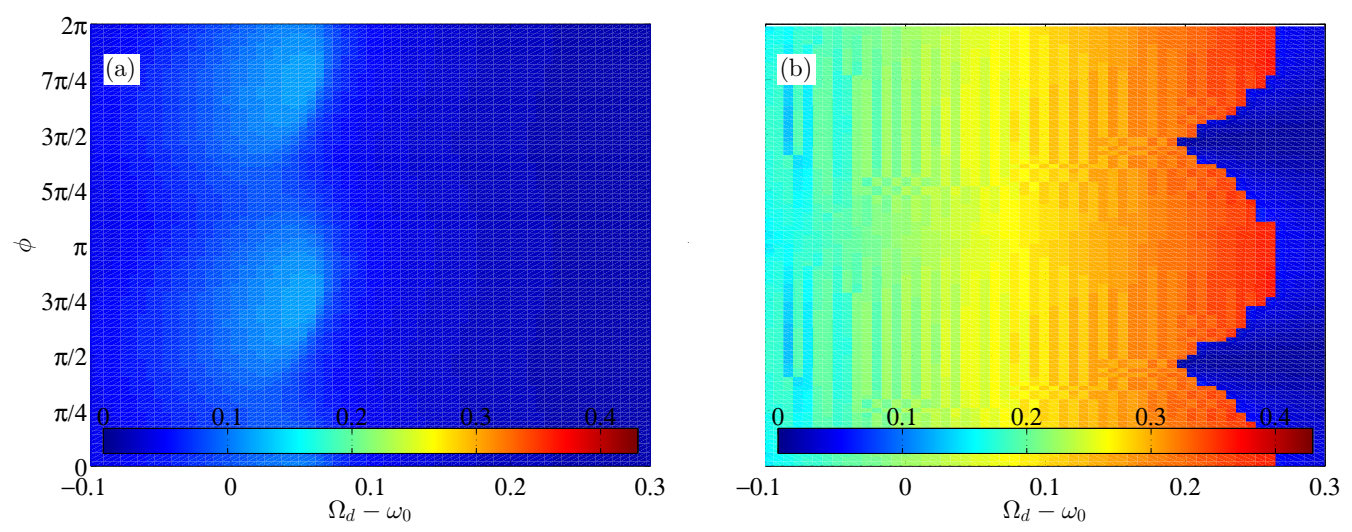

Figure 6: Numerical simulation of perfectly tuned (a) subthreshold $p=0.04$ and (b) superthreshold $p=0.8$ steady-state vibration amplitudes as function of excitation phase and direct excitation detuning. $(\gamma=10$. $)$

pendent variable, [10]. These results are discussed and compared with experimental results in Sect. 3.

\subsection{Summary}

The theoretical part shows good agreement between approximate analytical responses and results of direct numerical integration, both in the perfectly tuned and detuned cases, even for stronger nonlinearity and parametric excitation. The approximate analytical expression Eq. (16) provides responses 
which can be otherwise challenging to find numerically, and it can provide expressions for the backbones by zeroing the damping and external forces. Increasing the positive cubic nonlinearity makes the superthreshold parametric pumping response more hardened, just as in the classical case with subthreshold parametric pumping. For detuned superthreshold parametric pumping some of the amplitude-frequency branches appear to collapse, the frequency separation between the two peaks changes, and jumps are observed both during a down- and upsweep of the direct excitation frequency.

\section{Experimental observations}

Eq. (1) can model e.g. transverse vibration amplitudes of a cantilever beam. A cantilever beam is used as the model object with parametric amplification realized by adding direct and parametric excitation at the base. The base excitation components for a cantilever beam being directly and parametrically excited [10] have been simplified in Eq. (1) to focus on qualitative and not quantitative aspects. Experimental vibration amplitudes refer to the absolute maximum steady-state vibration amplitude scaled by the cantilever beam length.

\subsection{Setup}

Fig. 7 shows the experimental setup. A steel cantilever beam with length $\times$ width $\times$ thickness $=80 \times 8.5 \times 0.2 \mathrm{~mm}$, a fundamental natural frequency of $28.1 \mathrm{~Hz}$, and Young's modulus of approximately $210 \mathrm{GPa}$, is fixed at a B\&K Measurement Exciter Type 4808, with an angle between the imposed baseexcitation and the axial direction of the cantilever beam of 75 degrees. The signal to the vibration exciter is generated by an Agilent 33512B Waveform Generator and amplified by a B\&K Power Amplifier Type 2712. The transverse deflection of the cantilever beam is measured by an Omron ZX-LD40 Smart Sensor (laser displacement sensor) at the free end of the cantilever beam, digitally sampled and filtered using a National Instruments USB-6216 data acquisition module, and imported into National Instruments LabVIEW System Design Software for data processing. The output motion of the vibration exciter is checked using accelerometers at its base. The damping ratio for the cantilever beams lowest mode is about $0.1 \%$, estimated using standard impulse hammer testing. This is significantly lower than the damping ratio used in the theoretical predictions (10\%), which was chosen to reduce computational time to reach steady-state. Lowering the theoretical damping 


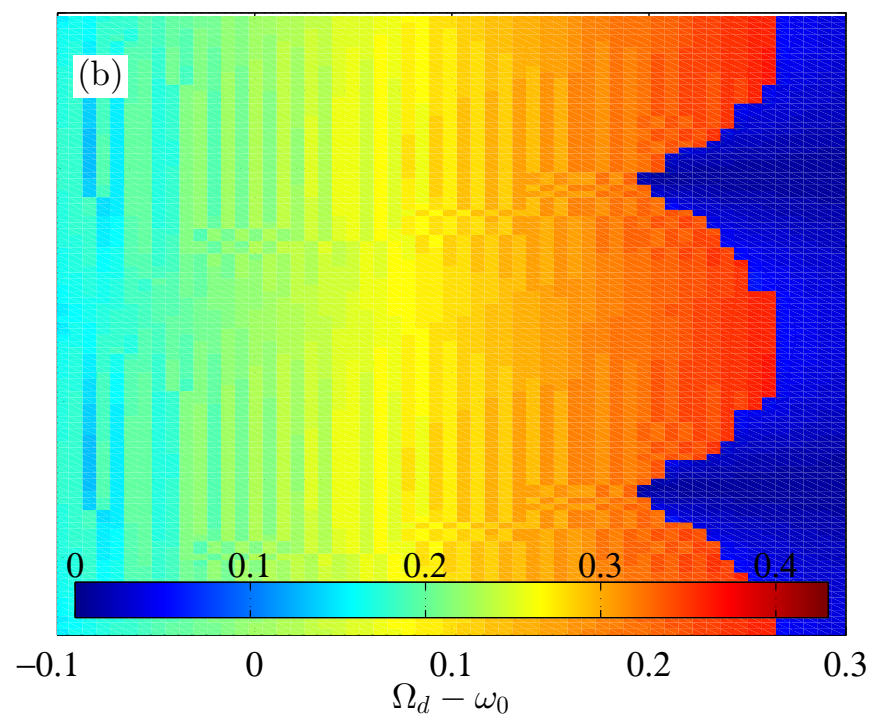

Figure 7: Experimental setup with custom made fixture attached to vibration exciter.

ratio to have the same damping ratio in the theoretical and experimental cases will alter the response quantitatively, mostly around the resonance frequency, but not qualitatively, neither in the case of subthreshold nor superthreshold parametric pumping. Thus, having different damping ratios in the theoretical and experimental cases are of minor importance as for testing the theoretical findings with regard to main parameter dependencies.

\subsection{Sub- and superthreshold parametrically pumped steady-state response}

Fig. 8 shows typical superthreshold displacement-time responses. Fig. 8(a) shows the response with perfect frequency tuning, whereas Fig. 8(b) shows the response with frequency detuning. Beating is observed in the detuned case. Qualitatively similar responses were obtained theoretically (inserts in Fig. 3).

Fig. 9 depicts the parametric instability threshold as function of parametric frequency detuning and parametric excitation amplitude (zero direct excitation). The parametric instability threshold appears as a sharp transition between a lower and higher steady-state vibration amplitude. For experimental subthreshold parametric excitation, the amplitude is chosen well below the parametric instability threshold, and for superthreshold parametric excitation the amplitude is chosen well above the parametric instability 

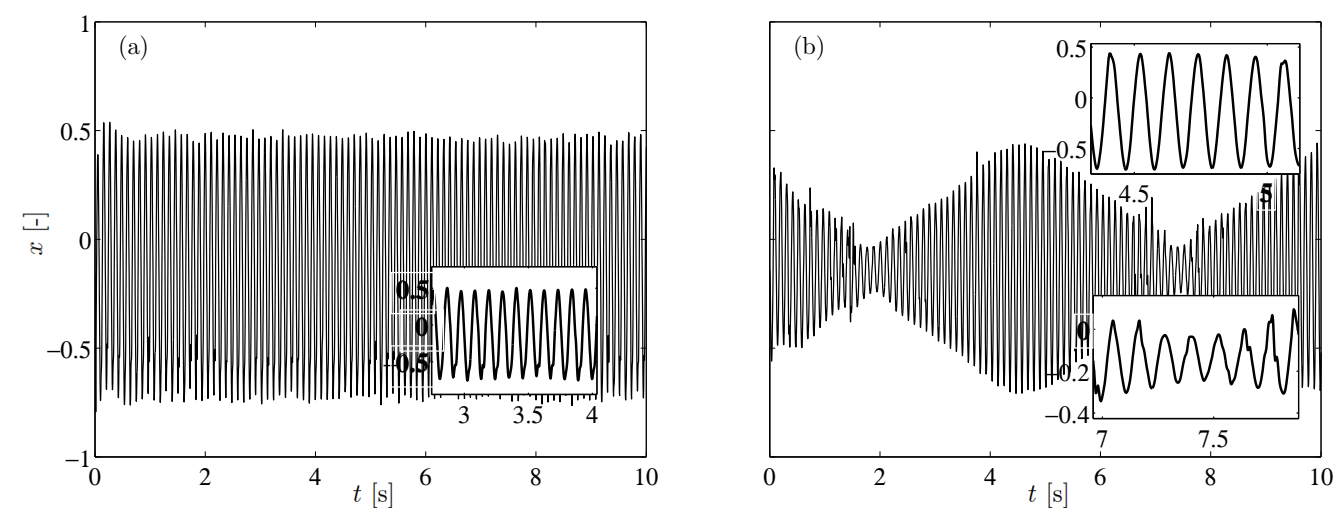

Figure 8: Experimental superthreshold displacement-time responses for (a) $\Omega_{p} / \Omega_{d}=2$ and (b) $\Omega_{p} / \Omega_{d}=2.05$. Inserts are blow ups.

\section{threshold.}

Fig. 10 shows a perfectly tuned amplitude-frequency response, obtained with a frequency up- and downsweep. A classical hardening (i.e. right bend) nonlinear resonance peak is observed. Notice the lower stable solutions on the peak; they appear to resemble the lower stable theoretical solutions on the peak in Fig. 2 (insert). The upper stable solutions on the peak might be obtained with a frequency upsweep with smaller increments; this is left for future studies. To the authors knowledge these experimental bistable amplified steady-state responses are the first reported in the literature. In the subsequent frequency experiments, both up- and downsweeps are conducted for the parametric and direct excitation frequencies. The direct excitation frequency is incremented in an inner sweep and the parametric excitation frequency in an outer sweep, unless otherwise stated.

Fig. 11 depicts experimental sub- (left) and superthreshold (right) parametrically pumped steady-state vibration amplitudes as function of parametric and direct excitation frequencies. Fig. 11(a) shows results for variation in direct and subthreshold parametric pumping. The maximum steady-state vibration amplitude is mostly sensitive to variation in direct excitation frequency. A notch is observed (the area entangled by dashed lines). For some detuning ranges, specifically within the notches, the lower solution below the peak seems to be preferred. These solutions are not results of instantaneous jumps, but results of beating, causing time intervals where the beam oscillates with a very small deflection around its equilibrium position, i.e. 


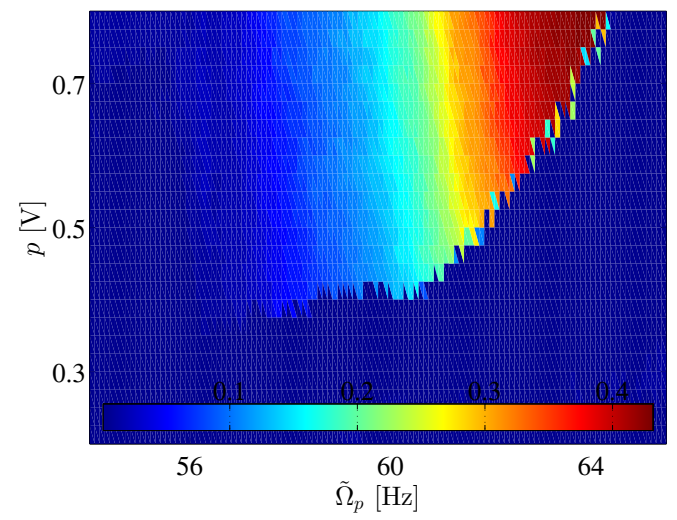

Figure 9: Experimental parametric instability threshold for the cantilever beam.

where an approximate zero amplitude is realized, and where the amplitude subsequently stays at approximately zero. The maximum steady-state vibration amplitude is, in the perfectly tuned case, located along the black solid line $\left(\Omega_{p} \approx 2 \Omega_{d}\right)$. Fig. $11(\mathrm{~b})$ shows results for variation in direct and superthreshold parametric pumping. An approximate two-to-one ratio between the parametric and the direct excitation frequency is denoted by the lower right black solid line. The amplitude-frequency relation corresponding to this line resembles results of Fig. 10, which is qualitative similar to the theoretical predictions (Fig. 2). Another notch appears as compared to the case with subthreshold parametric pumping. In these areas three stable solutions exist: an upper and a lower solution on the peak, and a lower solution below the peak. As discussed with Fig. 10, it seems that the lower solution on the peak is easier to obtain than the corresponding upper solution on the peak. The areas of these notches are considerably larger with superthreshold parametric pumping than with subthreshold pumping (compare figs. 11(a,b)). Within the areas of the notches related to superthreshold parametric pumping, one is capable of reaching the lower stable solution of the peak, as denoted by the few nonzero experimental results left of the markers I, II, and III, creating what could resemble three different horizontal lines. These results are similar to the lower stable response within the red square shown in Fig. 10. The frequency range with a steady-state vibration amplitude $a \geq 0.2$ increases in the case of superthreshold parametric pumping, but the emergence of an extra notch means that there exist one additional parameter subspace within which a smaller steady-state vibration amplitude (either the lower stable so- 


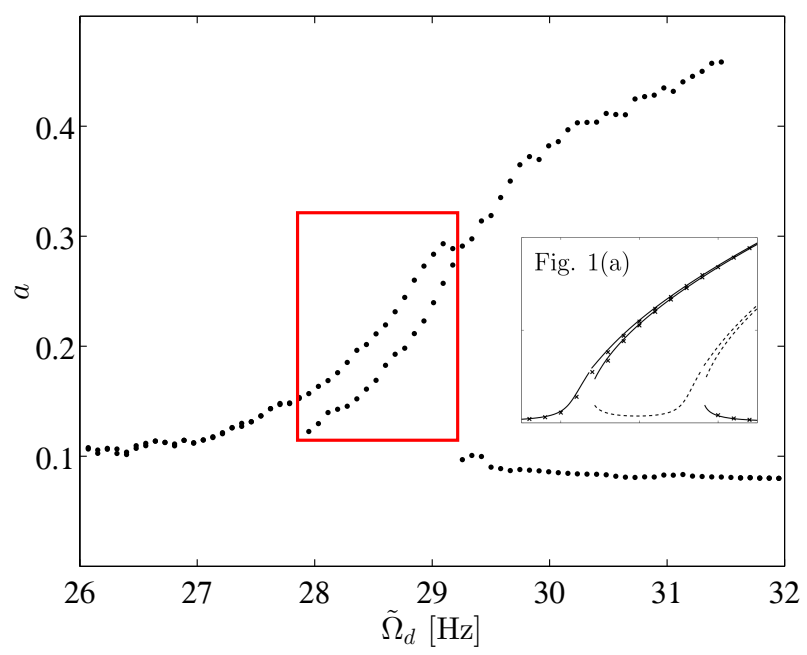

Figure 10: Experimental bistable amplified amplitude-frequency response. Insert is Fig. $2(\mathrm{a})$.

lution on the peak or the lower solution below the peak) is obtained. A suggested strategy, to look for these theoretical predictions experimentally, is to provoke a jump from the lower to the upper stable solution branch of the peak through a slowly increasing steady-state vibration amplitude, i.e. by increasing $p$ gradually, at a fixed frequency ratio and at a fixed closeness to resonance. This jump could occur when the attraction to the upper stable solution on the peak becomes stronger than the attraction towards the lower stable solution on the peak. The frequency range for which this is suggested, is as close to the lower fold point as possible, since the distance between the peaks upper and lower stable solution is largest here. One can also change this distance via the excitation phase. The theoretical predictions presented in Fig. 4 depict two special cases of the superthreshold parametric pumping experimental observations presented in Fig. 11. That is, figs. 4(a,b) are simplified representations of Fig. 11(b), namely for specific frequency ratios. One of these, for $\Omega_{p} / \Omega_{d}=1.95$, corresponds to the upper straight black line. Good agreement is noted: Jumps and the overall qualitative behaviour appears similar, and the change in frequency separation between the two peaks as predicted theoretically (Fig. 4) is also observed experimentally. Figs. 11(c,d) show that interchanging the order of parameter variation, i.e. fixing the parametric while varying the direct excitation frequency, results in an amplitude jump (red line in Fig. 11(d)) approximately at the lower left 

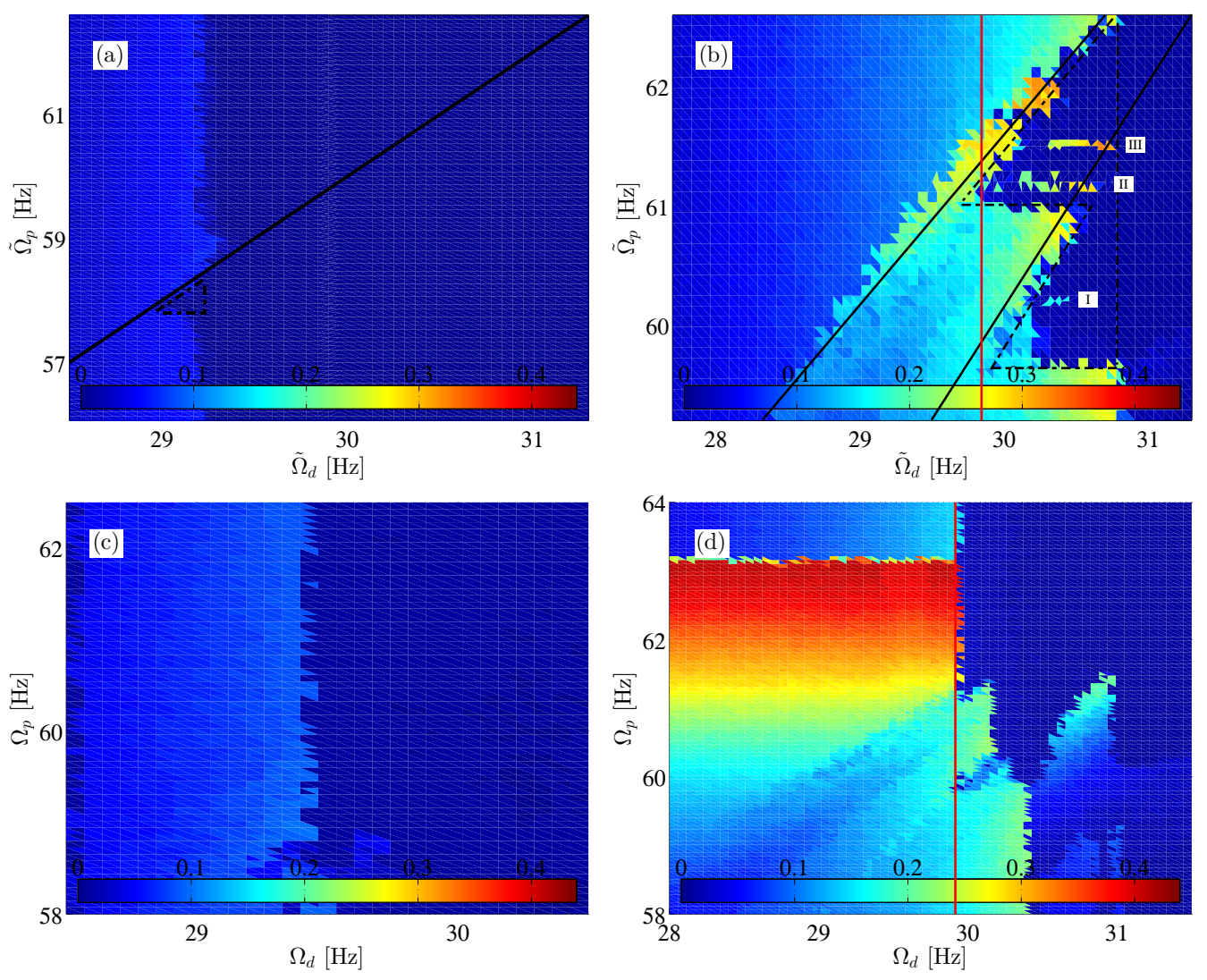

Figure 11: Experimental (a) sub- and (b) superthreshold parametrically pumped steadystate vibration amplitudes as a function of parametric and direct excitation frequencies, using a downsweep with $\tilde{\Omega}_{d}$ fixed while $\tilde{\Omega}_{p}$ is varied $(\mathrm{a}, \mathrm{b})$ and vice versa $(\mathrm{c}, \mathrm{d})$.

points of the notches in the initial results (Fig. 11(b)). The amplitude jump is related to parametric resonance, but the system is also subjected to direct excitation, which in the present case decreases the amplitude (the experimentally obtained steady-state vibration amplitude is normalized with respect to pure parametric resonance, which yields a higher steady-state amplitude in this study). Within the notch subspace, one is able to perturb the beam such that the lower solution of the peak is reached, but as in the case with fixed parametric excitation and varying direct excitation, the lower solution below the peak seems preferred (Fig. 10). This means that with changes in frequency at near-resonant oscillations, one can expect severe performance degradation for e.g. energy harvesters and sensors. In particular, one should 
account for performance degradation when an additional notch appears and small response amplitudes are obtained. Outside the frequency region of the notches, detuning of either the direct or parametric excitation frequency has a relatively small influence on the response.

In summary, frequency detuning can result in decreased as well as increased steady-state vibration amplitudes, making it an effect which could either be avoided or utilized dependent on the applications.

\subsection{Perfectly tuned case: dependency on the excitation phase}

Fig. 12 depicts sub- and superthreshold parametrically pumped steadystate vibration amplitudes as a function of excitation phase and direct excitation frequencies. A comparison with Fig. 6(b) shows that there appears to be an excitation phase lag of $\pi / 2$ between the theoretically and experimentally obtained (minimum and maximum) results. This offset is of no importance to the qualitative discussions. Fig. 12(a) shows the subthreshold parametrically pumped case. Typical jumps occur due to hardening, and a steady-state vibration amplitude which is somewhat dependent on the excitation phase is observed around resonance. The latter has also been observed in e.g. [10, 11]. This dependency on excitation phase diminishes with detuning from resonance. Fig. 12(b) shows that with superthreshold parametric pumping, a response with less dependency on the excitation phase is observed, and that the amplitude-excitation phase relationship becomes increasingly asymmetric for larger amplitudes (compare the black dotted lines in figs. $12(\mathrm{a}, \mathrm{b}))$. The steady-state vibration amplitudes are generally larger, and the jumps happen at larger direct excitation frequencies. Thus, the location of the jump between a higher and lower solution shifts as a function of the nonlinearity.

\subsection{Summary}

Experiments show that a drop in steady-state vibration amplitude occurs for specific areas in the amplitude-excitation detuning domain, indicating performance degradation, whereas for other parts of this domain an increased steady-state vibration amplitude can be obtained. The identified notches are not results of jumps, but reflect that beating causes moments where an approximate zero amplitude is realized, and which subsequently stays at approximately zero. With superthreshold parametric pumping the dependency on excitation detuning becomes less pronounced, as compared 

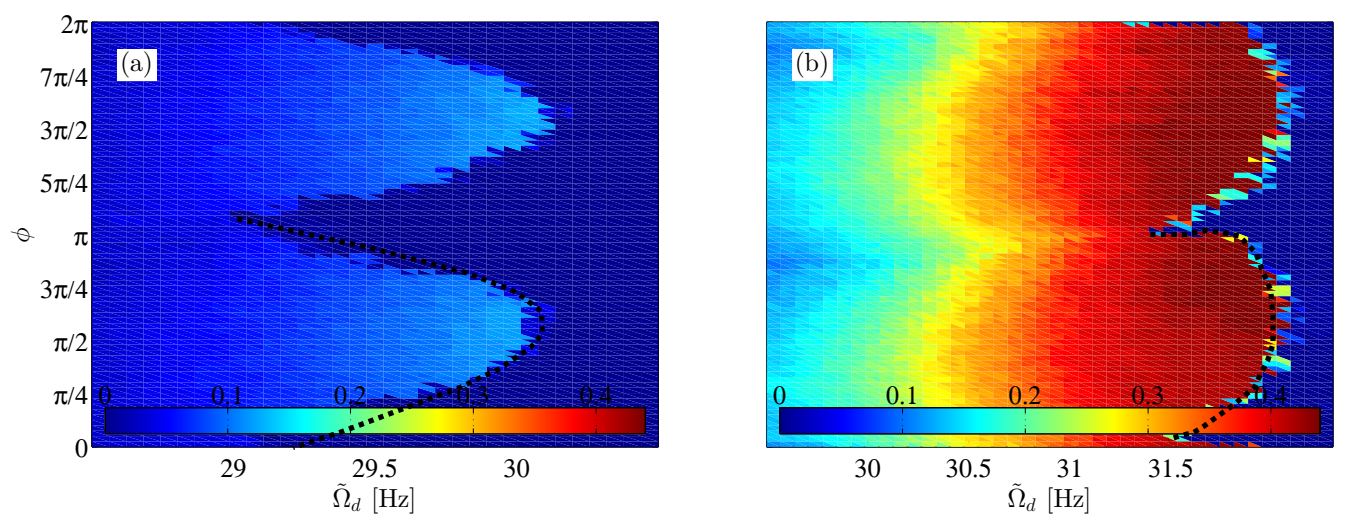

Figure 12: Experimental perfectly tuned (a) sub- and (b) superthreshold parametrically pumped steady-state vibration amplitudes as function of excitation phase and direct excitation frequency.

to subthreshold parametric pumping. Also, the steady-state vibration amplitudes are generally larger, and the jumps happen at larger direct excitation frequencies.

\section{Conclusions}

Frequency detuning effects between direct and parametric excitation frequencies for a parametric amplifier are investigated. The study is conducted theoretically using a Duffing-Mathieu equation as the model system, and experimentally using a macro cantilever beam as the model object. The approximate analytical steady-state vibration amplitudes are derived using the method of varying amplitudes and compared to results of direct numerical integration, showing good agreement both in the perfectly tuned and detuned case. For detuned superthreshold parametric pumping some of the theoretical amplitude-frequency curves appear to collapse, causing fewer potential jumps during operation. Qualitative agreement are generally obtained between the theoretical predictions, which are valid for a relatively wide range of system parameters, and experimental observations. Experiments show that a drop in steady-state vibration amplitude occurs for specific areas in the amplitude-excitation detuning domain indicating performance degradation, whereas for other parts of this domain an increased steady-state vibration amplitude can be obtained. This makes frequency detuning a feature which can purposefully be avoided or utilized, dependent on the application, e.g. 
with sensors and energy harvesters. Experimental bistable amplified steadystate responses are also demonstrated.

\section{Acknowledgement}

The authors thank Jan Justesen, Benny Edelsten, and Frida Husmark for their assistance with data processing equipment for the experimental setup.

\section{References}

[1] D. Rugar, P. Grütter, Mechanical parametric amplification and thermomechanical noise squeezing, Physical Review Letters 67 (1991) 699-702.

[2] M. Cartmell, Introduction to Linear, Parametric, and Nonlinear Vibrations, Chapman and Hall, London, 1990.

[3] B. Yurke, M. Roukes, R. Movshovich, A. Pargellis, A low-noise series-array josephson junction parametric amplifier, Applied Physics Letters 69 (1996) 3078-3080.

[4] R. A. Baumgartner, R. L. Byer, Optical parametric amplification, IEEE Journal of Quantum Electronics 15 (1979) 432-444.

[5] G. Mansfeld, N. Polzikova, I. Prokhorova, A. Raevskii, Parametric effects in composite microwave bulk-acoustic-wave resonators, Journal of Communications Technology and Electronics 48 (2003) 791-798.

[6] T. Ono, H. Wakamatsu, M. Esashi, Parametrically amplified thermal resonant sensor with pseudo-cooling effect, Journal of Micromechanics and Microengineering 15 (2005) 2282-2288.

[7] J. F. Rhoads, S. W. Shaw, K. L. Turner, Nonlinear dynamics and its applications in micro- and nanoresonators, Journal of Dynamic Systems, Measurement, and Control 132 (2010) 034001.

[8] R. Lifshitz, M. C. Cross, Nonlinear Dynamics of Nanomechanical and Micromechanical Resonators, Wiley-VCH, 2009.

[9] M. F. Daqaq, D. Bode, Exploring the parametric amplification phenomenon for energy harvesting, Proceedings of the Institution of Mechanical Engineers, Part I: Journal of Systems and Control Engineering 225 (2011) 456-466.

[10] J. F. Rhoads, N. J. Miller, S. W. Shaw, B. F. Feeny, Mechanical domain parametric amplification, Journal of Vibration and Acoustics 130 (2008) 061006.17.

[11] V. Kumar, J. K. Miller, J. F. Rhoads, Nonlinear parametric amplification and attenuation in a base-excited cantilever beam, Journal of Sound and Vibration 330 (2011) $5401-5409$. 
[12] J. F. Rhoads, S. W. Shaw, The impact of nonlinearity on degenerate parametric amplifiers, Applied Physics Letters 96 (2010) 234101.

[13] S. Neumeyer, J. J. Thomsen, Macroscale mechanical domain parametric amplification: superthreshold pumping and optimal excitation parameters, in: Proceedings of the EUROMECH $532-1^{\text {st }}$ International Colloquium on Time-Periodic Systems Current Trends in Theory and Application, Darmstadt, Germany, 2012, pp. 63-66.

[14] V. Sorokin, On the unlimited gain of a nonlinear parametric amplifier, Mechanics Research Communications 62 (2014) 111-116.

[15] S. Neumeyer, J. J. Thomsen, Macromechanical parametric amplification with a baseexcited doubly clamped beam, in: Proceedings of $11^{\text {th }}$ International Conference on Vibration Problems, ICOVP, Lisbon, Portugal, 2013. 8pp.

[16] S. Neumeyer, R. V. D. Looij, J. J. Thomsen, Jumps and bi-stability in the phase-gain characteristics of a nonlinear parametric amplifier, in: Proceedings of $8^{\text {th }}$ European Nonlinear Dynamics Conference, ENOC, Vienna, Austria, 2014. 2pp.

[17] V. Ramakrishnan, B. F. Feeny, Resonances of a forced mathieu equation with reference to wind turbine blades, Journal of Vibration and Acoustics 134(6) (2012) 064501.

[18] S. Neumeyer, V. Sorokin, J. Thomsen, Effects of quadratic and cubic nonlinearities on a perfectly tuned parametric amplifier, Journal of Sound and Vibration 386 (2016) $327-335$.

[19] Y. Jia, S. Du, A. A. Seshia, Twenty-eight orders of parametric resonance in a microelectromechanical device for multi-band vibration energy harvesting, Scientific Reports 6 (2016) 30167.

[20] V. S. Sorokin, J. J. Thomsen, Vibration suppression for strings with distributed loading using spatial cross-section modulation, Journal of Sound and Vibration 335 (2015) 66-77.

[21] S. Neumeyer, M. H. M. v. Gastel, V. S. Sorokin, J. J. Thomsen, Frequency detuning effects for parametrically and directly excited elastic structures, in: Proceedings of $5^{\text {th }}$ International Conference on Computational Methods in Structural Dynamics and Earthquake Engineering, COMPDYN, Crete Island, Greece, 2015. 7pp.

[22] A. H. Nayfeh, D. T. Mook, Nonlinear Oscillations, Wiley, New York, 1979.

[23] C. Kim, C. Lee, N. Perkins, Nonlinear vibration of sheet metal plates under interacting parametric and external excitation during manufacturing, Journal of Vibration and Acoustics 127 (2005) 36-43.

[24] D. Zulli, A. Luongo, Bifurcation and stability of a two-tower system under windinduced parametric, external and self-excitation, Journal of Sound and Vibration 331 (2012) 365-383. 